\title{
Towards the standardization of fullerene nanofibers using Raman spectroscopy
} \author{
Bonifacio $^{6}$, Froylan Martinez ${ }^{7}$, Ryoei Kato ${ }^{8}$ and Kun'ichi Miyazawa ${ }^{8}$ \\ ${ }^{1}$ Inmetro, Av. Nossa Senhora das Graças, 50, Duque de Caxias, RJ, Brazil \\ ${ }^{2}$ National Institute of Metrology, No.18,Bei San Huan Dong Lu, Beijing, China \\ ${ }^{3}$ Qingdao University of Science and Technology, 99 Songling Rd, Laoshan, Qingdao, China \\ ${ }^{4}$ Korea Advanced Institute of Science and Technology, 373-1 Guseong-dong, Yuseong-gu, Daejon, Korea \\ ${ }^{5}$ Yokohama City University, 22-2 Seto, Kanazawa-ku, Yokohama, Japan \\ ${ }^{6}$ University of Trieste, Piazzale Europa, 1, Trieste, Italy \\ ${ }^{7}$ National Metrology Center, km 4.5 Carretera a Los Cués, Querétaro, Mexico \\ ${ }^{8}$ National Institute for Materials Science, 1-1, Namiki, Tsukuba, Japan
}

Erlon H Martins Ferreira ${ }^{1, \mathrm{a}}$, Lingling Ren ${ }^{2}$, Guangzhe Piao ${ }^{3}$, Soon Hyung Hong ${ }^{4}$, Masaru Tachibana ${ }^{5}$, Valter Sergo ${ }^{6}$, Alois

\begin{abstract}
Résumé. Le spectre Raman de nanofibres de fullerène a été acquis avec différentes densités de puissance laser incidentes afin d'évaluer son influence sur la position du pic du mode $\mathrm{A}_{\mathrm{g}}(2)$ de la molécule de fullerène. Une procédure pour mesurer le diamètre du faisceau laser et la densité de puissance sur l'échantillon dans un microscope Raman est proposé. Les incertitudes du processus de mesure et d'étalonnage du spectromètre sont également présentés.
\end{abstract}

\section{Introduction}

Fullerene nanofibers are defined as the fibers that are composed of fullerene molecules and have diameters less than $1000 \mathrm{~nm}[1,2]$. The fullerene nanofibers can take both the non-tubular and tubular morphologies. The nontubular fullerene nanofiber with single crystalline structure is called "fullerene nanowhisker", while the tubular fullerene nanofiber is called "fullerene nanotube". The fullerene nanofibers are the new semiconducting materials that are applicable to solar cells, templates for chemical synthesis, MEMS cantilever beams, field-effect transistors, catalysts, composite fillers, superconductors and so on. The researcher population of fullerene nanofibers is small at the present time. However, it is gradually increasing, and will increase more with the decreasing price of fullerenes. Hence, the prestandardization is necessary for future popularization of fullerene nanofibers.

The interlaboratory comparison of Raman spectroscopy data of Fullerene nanofibers has been established inside the TWA 34 of VAMAS (Versailles Project on Advanced Materials and Standards). The goal of the project is to determine the optimum parameters of exposure power density and time, sample preparation protocol, and evaluation of Raman shift uncertainties. Two round-robins have been performed and the Raman profiles have been obtained in different conditions by different laboratories. It has been found a large scattering

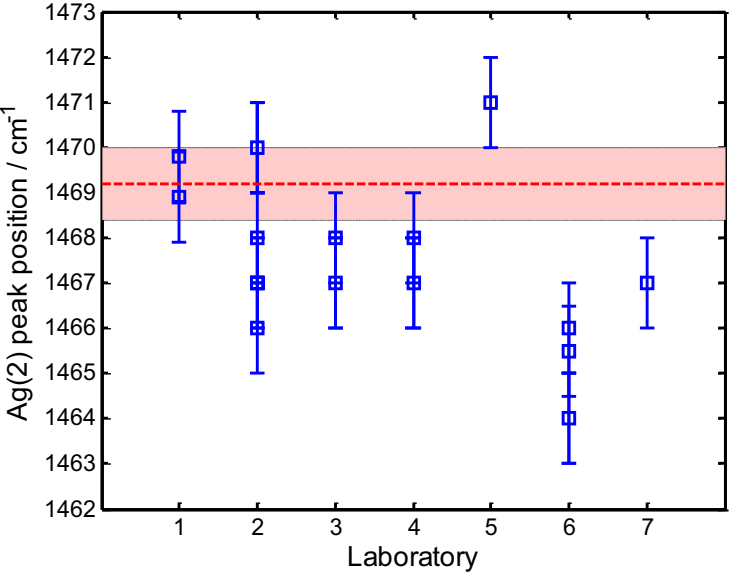

Figure 1. $\operatorname{Ag}(2)$ peak of fullerene nanofiber measured by different laboratories in a first round robin. The red line is the expected value.

in the reported data, and part of this effect can be attributed to the photopolymerization of the fullerene molecules (see Fig.1).

Not long after the discovery of the fullerene molecules by Kroto, Smalley and co-workers[3] in the middle 1980's, people started soon investigating their properties and looking for new structures formed with those molecules. In the beginning of 1990's, several works[4-7] reported the effect of light on the crystalline

\footnotetext{
${ }^{\text {a }}$ Corresponding author: ehferreira@inmetro.gov.br
} 
structure of fullerene films, causing a polymerization of the molecules (photopolymerization). It has been observed that this effect occurs only for UV-visible light, where the energy of photon is greater than optical absorption edge at $1.6 \mathrm{eV}$, and for some conditions of power/energy densities of incident radiation. The same effect has also been reported for fullerene nanofibers[8]. There is, however, no detailed discussion on the threshold values of power/energy densities for different excitation sources.

In this work, we have used the Raman $A_{g}(2)$ peak position of fullerene nanofibers and fullerene crystals to study the photopolymerization effect caused by the variation of incident laser power density. We seek to fulfil the detailed information needed for the Raman measurement of fullerene nanofibers in a normative documentary standard.

\section{Materials and methods}

A $\mathrm{C}_{60}$-saturated toluene solution was prepared by dissolving $\mathrm{C}_{60}$ powder (99.5\% MTR Ltd., USA) in toluene (non-dehydrated, Wako Pure Chemical Industries, Ltd., Japan). The $\mathrm{C}_{60}$ nanofibers (nanowhiskers) were synthesized through a modified liquid-liquid interfacial precipitation method (LLIP) method [9]. A $\mathrm{C}_{60}$-saturated toluene solution was poured into a glass bottle and an equal amount of isopropyl alcohol (IPA) was layered onto the $\mathrm{C}_{60}$-saturated toluene solution. The glass bottle was capped and manually mixed by shaking the bottle 10 times and stored in an incubator at $15^{\circ} \mathrm{C}$ for 5 days to obtain the $\mathrm{C}_{60}$ nanowhiskers with a mean length of $12.0 \mu \mathrm{m}$.

To make the Raman measurements, the dried fullerene nanofibers was dispersed in isopropyl alcohol, dropped in a glass slide and let to dry in air

Raman measurements were performed using a Renishaw Invia spectrometer, in backscattering configuration using a $50 \mathrm{x}$ microscope objective (NA= $0.75)$. The excitation light was the green line $(514.5 \mathrm{~nm})$ of an $\mathrm{Ar} / \mathrm{Kr}$ laser. The laser beam is a Gaussian shaped and its full width at half maximum has been measured using the procedure describe in section 3.2.

The laser power at the sample was measured using a laser power meter, and it was varied from approximately $1 \mu \mathrm{W}$ to $300 \mu \mathrm{W}$ using a combination of different neutral density filters.

For each power density, five spectra were acquired at different points of the sample. The acquisition time was set in order to keep the total incident laser energy around $60 \mathrm{~J} / \mathrm{mm}^{2}$ in every measurement.

\section{Determination of the laser power density}

Seeking for standardization, it is very important that the measurements have a high degree of confidence. A critical parameter in the analysis of the fullerene nanofibers is the power density at the sample, as fullerene is sensitive to light exposure, and may go through a polymerization process.

The power density, $P D$, at the sample is calculated as

$$
P D=\frac{4 P_{0}}{\pi D^{2}}
$$

where $P_{0}$ is the measured power, and $D$ is the laser beam diameter. The measurement of $D$ is not trivial however.

In Gaussian beam optics, $D$ can be calculated considering it equal to the beam waist at the focus. For an uniformly illuminated objective, the diffraction pattern is the classical Airy disk, with a central bright spot and following weaker rings. The diameter of the central spot, i.e., twice the distance from the centre to the first dark ring, is given by

$$
D=\frac{1.22 \lambda}{\mathrm{NA}}
$$

Here, $\lambda$ is the light wavelength and NA is the numerical aperture of the microscope objective. Equation (2) is the theoretical value, and holds for a perfect aligned optics. This is however rarely the case for a multiuser routine equipment. In this case, one shall find a method of measuring the laser beam profile.

\subsection{Laser beam profile}

A laser beam used in Raman spectroscopy has a profile that is typically Gaussian. Considering a Gaussian beam with standard deviation $\sigma$, the intensity distribution can be described by

$$
I(x, y)=\frac{P_{0}}{2 \pi \sigma^{2}} \exp \left[-\left(x^{2}+y^{2}\right) / 2 \sigma^{2}\right],
$$

where $P_{0}$ is total power (integrated intensity) of the beam.

As the beam intensity is not uniformly distributed, $P D$ depends on the definition of the beam diameter. Typically, we can assume $D$ to be equal the distance where the intensity of the beam falls to $1 / \mathrm{e}^{2}$. This is equivalent to two standard deviation $(r=2 \sigma$ or $D=4 \sigma)$, and the integrated intensity at this point is $\sim 0.86 P_{0} . P D$ calculated using this definition of $D$ is called "mean power density" and given by

$$
\overline{P D}=\frac{\left(1-\mathrm{e}^{-2}\right) P_{0}}{4 \pi \sigma^{2}}=0.3816 \frac{P_{0}}{\mathrm{FWHM}^{2}} .
$$

The full width at half maximum, FWHM, of a Gaussian distribution is equal to $2.35482 \sigma$.

Another parameter that can also be used is the maximum power density, calculated at the centre of the beam distribution. This is given by

$$
\operatorname{MaxPD}=\frac{P_{0}}{2 \pi \sigma^{2}}=\frac{0.8825 P_{0}}{\mathrm{FWHM}^{2}} .
$$

\subsection{Measuring the FWHM of a laser beam}

There are several methods to measure a laser beam profile. A beam profiler can be used to measure the characteristics of a beam, but this is not suitable to use under a microscope objective of high magnification, which is normally used during Raman measurements. 


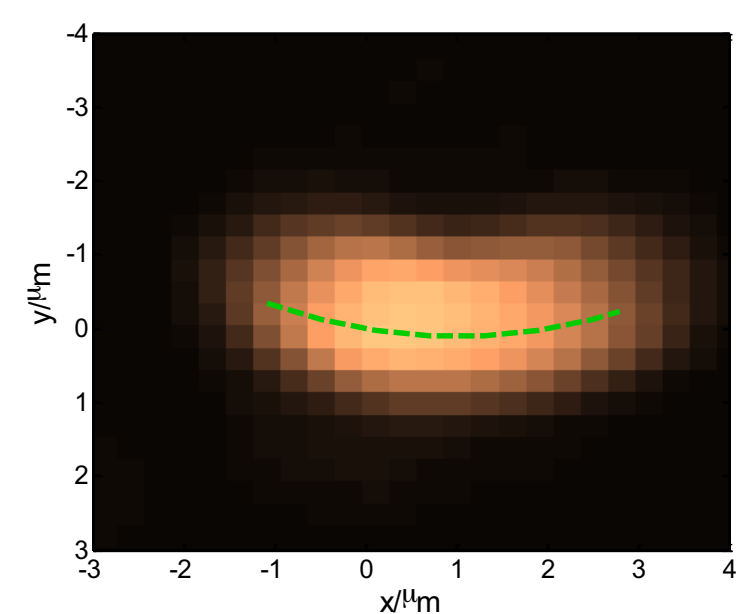

Figure 2. Raman map of the $\mathrm{G}$ band of a small bunch of carbon nanotubes. The size of bunch ( $20 \mathrm{~nm}$, indicated as the green line) is much smaller than the apparent size because of the size of the laser spot.

Another method has been developed to measure the laser beam profile. Single walled carbon nanotubes (SWCNT) have a high Raman cross-section and even a bunch with few tubes have a signal high enough to be easily detected. Scanning the laser beam across the tube, the Raman signal will be proportional to the integrated power in the direction of the tube. Since the diameter of the tube is much smaller than the FWHM of the beam, there is no interference of its size in the measurement.

A solution with SWCNT (IsoNanotubes-S, NanoIntegris, USA) was dropped onto a silicon substrate and a bunch of tubes was located with the help of an optical microscope. The diameter of the bunch was measured with AFM and found to be around $20 \mathrm{~nm}$, which is much smaller than the FWHM of the laser beam. A Raman map of this same bunch was then performed.

Figure 2 shows the Raman map of the integrated intensity of the $\mathrm{G}$ band of the nanotubes. This image shows that the apparent size of the bunch is much greater than the real size because of the laser beam width. The intensity profile across the $y$ direction has a Gaussian shape, which is the shape of the beam.

For each vertical scan line, the laser beam profile has been determined, and the FWHM was calculated using a fitting procedure. Figure 3 shows the measured values of the FWHM along different points of the tube. The mean

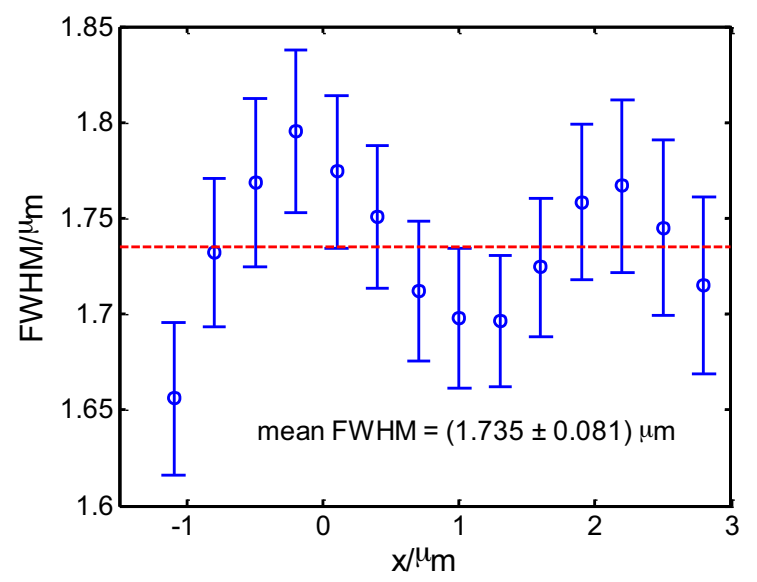

Figure 3. Full width at half maximum of the laser beam measure using the Raman signal of bunch of SWCNT as spike.
FWHM of laser beam is $(1.735 \pm 0.081) \mu \mathrm{m}$, with $k=1.96$, and level of confidence of $95 \%$.

\section{Spectral calibration}

One important aspect in Raman measurement is the spectrometer calibration. Before every measurement, a reference material is used to check the equipment calibration. In this work, we have used cyclohexane as reference, whose peak positions are tabulated in the ASTM E1840-96 (2014) standard.

Since the peak of interest is around $1470 \mathrm{~cm}^{-1}$, only the peaks between $800 \mathrm{~cm}^{-1}$ and $1444 \mathrm{~cm}^{-1}$ have been used in the calibration. The position of the cyclohexane peaks were found using a fitting procedure in order to minimize the uncertainty in the peak position. The measured values $M_{i}$ are plotted as a function of the reference values $R_{i}$, and a linear regression is performed, such that

$$
M_{i}=\alpha+\beta R_{i}+\varepsilon_{i}
$$

where $\varepsilon_{i}$ is the measurement error, $\alpha$ and $\beta$ are the coefficients of the regression.

The corrected value, $C$, of a measured peak position $P$ and the associated uncertainty $u_{\text {cal }}$ are simply

$$
\begin{gathered}
C=(P-\alpha) / \beta \\
u_{c a l}=\frac{\sigma}{\beta} \sqrt{\frac{1}{m}+\frac{1}{n}+\frac{(P-\bar{M})^{2}}{\beta \sum_{i=1}^{n}\left(R_{i}-\bar{R}\right)^{2}}} .
\end{gathered}
$$

Here $m$ is the number of observations of $P, n$ is the number of points in the calibration curve, $\sigma$ is the variance of the regression data, and a bar symbol $\left(^{-}\right)$ over a variable represents the mean.

\section{Raman measurement of the fullerene nanofiber and uncertainties evaluation}

The Raman spectrum of a fullerene nanofiber is the same of the fullerene crystal. The $\mathrm{C}_{60}$ molecule has 46 distinct intramolecular vibrational modes which correspond to the following symmetries[10]

$$
\begin{aligned}
\Gamma_{\text {vib }}=2 \mathrm{~A}_{\mathrm{g}}+3 \mathrm{~F}_{1 \mathrm{~g}}+4 \mathrm{~F}_{2 \mathrm{~g}}+ & 6 \mathrm{G}_{\mathrm{g}}+8 \mathrm{H}_{\mathrm{g}}+\mathrm{A}_{\mathrm{u}} \\
+ & 4 \mathrm{~F}_{1 \mathrm{u}}+5 \mathrm{~F}_{2 \mathrm{u}}+6 \mathrm{G}_{\mathrm{u}}+7 \mathrm{H}_{\mathrm{u}} .
\end{aligned}
$$

Only 10 out of the 46 mode frequencies are Raman active $\left(2 \mathrm{~A}_{\mathrm{g}}+8 \mathrm{H}_{\mathrm{g}}\right)$ and 4 are infrared active $\left(4 \mathrm{~F}_{1 \mathrm{u}}\right)$; the other modes are optically silent. Figure 4 shows a typical Raman spectrum of the fullerene nanofiber. The $2 \mathrm{~A}_{\mathrm{g}}$ peaks are the more intense and they are related with the radial breathing mode $\left(\mathrm{A}_{\mathrm{g}}(1)\right)$ and the "pentagonal pinch" mode $\left(\mathrm{A}_{\mathrm{g}}(2)\right)$. When the fullerene undergoes a polymerization, the $A_{g}(2)$ shifts to smaller frequency. The inset of Figure 4 compares a spectrum of nonpolymerized material (blue/dashed line) with the polymerized one (red/full line). We can observe also a broadening in the $\mathrm{Ag}_{\mathrm{g}}(2)$, which is an indication of the 


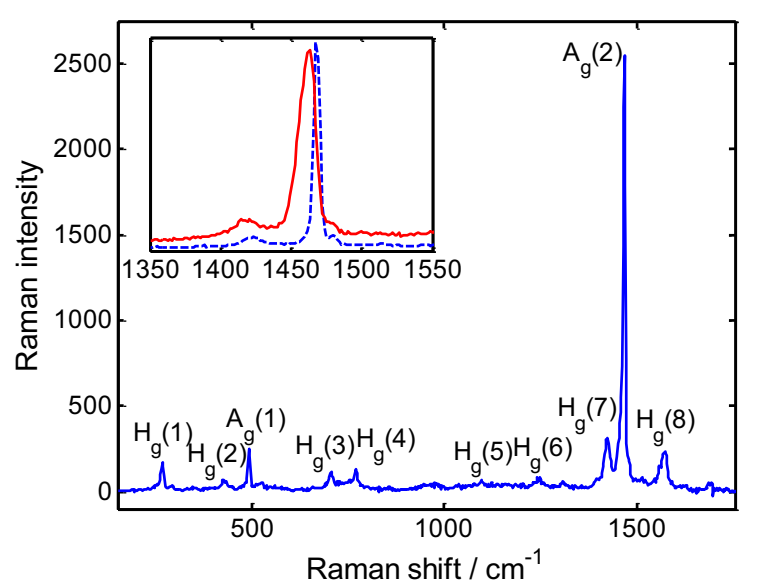

Figure 4. Typical Raman spectrum of a fullerene nanofiber showing the Raman active modes. The inset picture shows a zoom in the region of the $\mathrm{A}_{\mathrm{g}}(2)$ band, where the red line (full line) is for a polymerized $\mathrm{C}_{60}$ in comparison with the blue (dashed line) for a non-polymerized material.

presence of disorder in the crystalline structure due to the molecule polymerization.

In order to see how the incident laser power changes the Raman spectrum of the fullerene nanofibers, a set of measurements with different laser power was conducted. The measurement process was following:

1. The reference material (cyclohexane) is measured and the coefficients of the linear regression (Eq. 6) are found;

2. For each laser power condition, five Raman spectra are acquired at different points of the sample;

3. The $A_{g}(2)$ band of each spectrum is fitted using a Lorentzian curve and least square method;

4. The mean value of the $\mathrm{A}_{\mathrm{g}}(2)$ peak positions is calculated;

5. The calibration curve (Eq. 7) is used to correct the value of the peak position.

There are three main contributions to the uncertainty in the peak position measurement in this case:

1. The uncertainty of the fitting, $u_{\text {fit }}$;

2. The uncertainty of the mean, $u_{\text {std }}$;

3. The uncertainty of the calibration, $u_{\text {cal }}$.

The uncertainty of the fitting is the mean value of the uncertainties of each least square procedure, and have a degree of freedom that can be considered infinite; the uncertainty of the mean is calculated as the standard deviation of mean, and the degree of freedom is $m-1=$ 4; the uncertainty of the calibration is given by Eq. (9), and in this case the degree of freedom is $n-2=3$.

We calculate the combined uncertainty and the effective degree of freedom by

$$
\begin{gathered}
\left(u_{\mathrm{c}}\right)^{2}=\left(u_{\mathrm{fit}}\right)^{2}+\left(u_{\mathrm{std}}\right)^{2}+\left(u_{\mathrm{cal}}\right)^{2}, \\
v_{\text {eff }}=\left(u_{\mathrm{c}}\right)^{4} /\left[\left(u_{\mathrm{std}}\right)^{4} / 4+\left(u_{\mathrm{cal}}\right)^{4} / 3\right] .
\end{gathered}
$$

The expanded uncertainty is calculated as $U=k u_{\mathrm{c}}$, where $k$ is a coverage factor calculated for a level of confidence (or coverage probability, CP) of $95 \%$, based in a t-distribution.

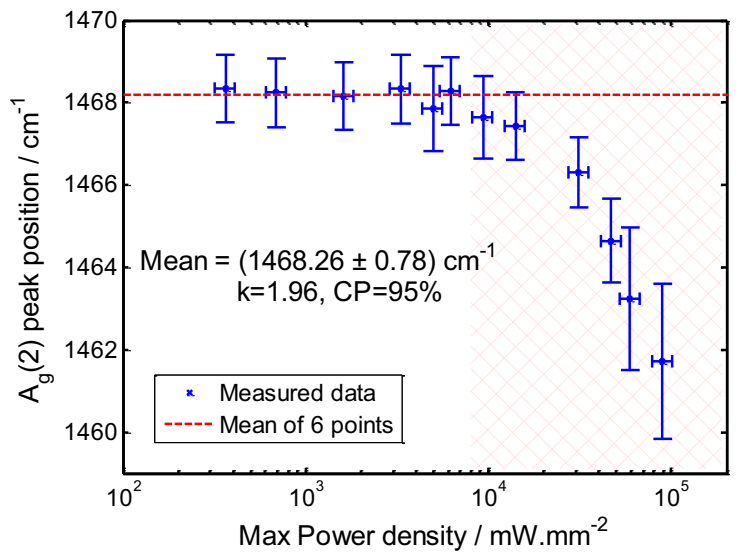

Figure 5. $\operatorname{Ag}(2)$ peak position of fullerene nanofiber as function of the laser power density. The red/dashed line represents the mean value of the peak position for the first six measurements, where we consider the fullerene is non-polymerized.

Figure 5 shows the results of the $\mathrm{A}_{\mathrm{g}}(2)$ peak position determination for different incident laser power. The maximum power density at the sample was calculated using Equation 5. We see that there is a clear threshold where the peak position starts to decrease (red crossed area in Figure 5) that happens around $9 \times 10^{3} \mathrm{~mW} / \mathrm{mm}^{2}$. If only the data below this threshold is considered, the mean value of the $\mathrm{A}_{\mathrm{g}}(2)$ peak position is $(1468.26 \pm 0.78)$ $\mathrm{cm}^{-1}$, with $k=1.96$ for a coverage probability of $95 \%$.

\section{Conclusions}

In this work we have presented a study of the influence of the laser power density in the Raman spectrum of fullerene nanofibers. The standardization process for the measurement is of vital importance in quality control. A method to estimate the laser beam diameter and the power density at the sample was proposed. The uncertainties of the measurement were calculated considering the repeatability, the fitting process error and the calibration.

It was found that for measurements with a maximum power density below $9 \times 10^{3} \mathrm{~mW} / \mathrm{mm}^{2}$, there is no indication of polymerization of the fullerene molecule in the Raman spectrum. This value should be considered as a limit when performing Raman measurements.

\section{References}

1. K. Miyazawa, A. Obayashi, and M. Kuwabara, J. Am. Ceram. Soc. 84, 3037-3039 (2001)

2. K. Miyazawa, Y. Kuwasaki, A. Obayashi and M. Kuwabara, J. Mater. Res. 17, 83-88 (2002)

3. H. W. Kroto, J. R. Heath, S. C. O'Brien, R. F. Curl, and R. E. Smalley, Nature, 318, 162-163 (1985)

4. P. Zhou, A. M. Rao, K. Wang, J. D. Robertson, C. Eloi, M. S. Meier, S. L. Ren, XiangXin Pi, P. C. Eklund, and M. S. Dresselhaus, Appl. Phys. Lett. 60, 2871-2873 (1992)

5. A. M. Rao, P. Zhou, K. Wang, G. T. Hager, J. M. Holden, Y. Wang, W. Lee, XiangXin Pi, P. C. 
Eklund, D. S. Cornett, M. A. Duncan, and I. J. Amster, Science 259, 955-957 (1993)

6. D. S. Cornett, I. J. Amster, M. A. Duncan, A. M. Rao, and P. C. Eklund, J. Phys. Chem. 97, 50365039 (1993)

7. M. S. Dresselhaus, G. Dresselhaus, and P. C. Eklund, "7.5 Polymerized Fullerenes" in: Science of Fullerenes and Carbon Nanotubes, 209-217 (Academic Press, San Diego, 1995)

8. M. Tachibana, K. Kobayashi, T. Uchida, K. Kojima, M. Tanimura, and K. Miyazawa, Chem. Phys. Lett. 374, 279-285 (2003)

9. K. Miyazawa, C. Hirata, T. Wakahara, Journal of Crystal Growth 405, 68-72 (2014)

10. H. Kuzmany, R. Pfeiffer, M. Hulman, and C. Kramberger, Phil. Trans. R. Soc. Lond. A 362, 2375-2406 (2004). 